\title{
EI Magisterio público de la ciudad de Santiago de Cuba: formación y superación entre 1899 a $1915^{1}$
}

\author{
Daineris Mancebo Céspedes ${ }^{2}$ \\ Universidad de Oriente, Cuba
}

Recepción: 26/09/2020

Evaluación: 30/09/2020

Aceptación: 15/10/2020

Artículo de Investigación-Científica

DOI: https://doi.org/10.22267/rhec.202525.80

\section{Resumen}

Desde el periodo de ocupación norteamericano en Cuba (1899-1902) constituyó una premisa para las fuerzas estadounidenses impulsar la instrucción pública en la Isla. Con tal fin, una de las primeras medidas que asumieron fue preparar a los hombres y mujeres que integrarían las filas del sector magisterial. Este artículo aborda cuáles fueron esos primeros pasos en la formación de los maestros a desempeñarse en la instrucción primaria, a través del territorio de Santiago de Cuba, en el periodo de 1899 a 1915. A través de la selección de una copiosa información, se acoge a la metodología de la investigación histórica. Se persigue que contribuya a continuar enriqueciendo la Historia de la Educación, como una de las líneas de investigación más concurridas por historiadores cubanos y de otras partes del mundo en las últimas décadas.

Palabras clave: Cuba; enseñanza pública; Escuela Normal; formación de docentes.

1 Este artículo se deriva de la investigación doctoral titulada "El magisterio público en la formación de la conciencia histórica, cívica y patriótica en la enseñanza primaria pública de la ciudad de Santiago de Cuba entre 1899 a 1915".

2 Profesora en el Departamento de Historia de la Universidad de Oriente, Cuba. Línea de investigación: Historia de la educación. Correo electrónico: daineris@uo.edu.cu. http://orcid.org./0000-0001-9669-0660 


\title{
Public school teachers from Santiago de Cuba: training and improvement from 1899 to 1915
}

\begin{abstract}
Since the period of U.S. occupation in Cuba (1899-1902), it was a premise for the U.S. forces to promote public education on the island. To this end, one of the first measures taken was to prepare the men and women who would join the positions of the teaching profession. This article deals with those first steps in the training of teachers to work in primary education, throughout the territory of Santiago de Cuba, from 1899 to 1915. Through the selection of detailed and abundant information, this academic paper uses the methodology of historical research. It is intended to contribute to continue enriching the History of Education, as one of the most popular lines of research by Cuban historians and from other parts of the world in the last few decades.
\end{abstract}

Keywords: Cuba; public education; Escuela Normal; teacher training.

\section{O Magistério público da cidade de Santiago de Cuba: formação e aperfeiçoamento entre 1899 e 1915}

\section{Resumo}

Desde o período da ocupação norte-americana em Cuba (1899-1902), constituiu uma premissa para as forças estadunidenses promoverem a educação pública na Ilha.Para isso, uma das primeiras medidas que tomaram foi a preparação de homens e mulheres que integraria as fileiras do setor de ensino. Este artigo aborda quais foram os primeiros passos na formação de professores para atuar no ensino fundamental, em todo o território de Santiago de Cuba, no período de 1899 a 1915. Mediante a 
seleção de uma abundante quantidade de informações, dá-se as boas-vindas a metodologia da pesquisa histórica. Pretende-se contribuir para continuar enriquecendo a História da Educação, como uma das linhas de pesquisa mais freqüentadas por historiadores cubanos e de outras partes do mundo nas últimas décadas.

Palavras-chave: Cuba; educação pública; escola normal; formação de professores.

\section{Introducción}

Una de las primeras acciones que se llevaron a cabo por las autoridades norteamericanas durante el periodo de ocupación de sus fuerzas en Cuba (1899-1902) fue atender la instrucción pública, debido a su deterioro por el impacto de largos años de lucha por la independencia. Precisamente, una de las primeras disposiciones que se establecieron fue formar a los hombres y mujeres que tendrían la misión de educar e instruir a los ciudadanos del mañana, especialmente desde las primeras edades, a través de la instrucción primaria pública.

La formación del magisterio que se iba a desempeñar en la instrucción primaria pública la ha abordado la historiografía cubana, como uno de los tópicos de la denominada Historia de la Educación. ${ }^{3}$ Ellos fueron los llamados "maestros de certificado", práctica que tuvo lugar en nuestro país hasta la institucionalización de la Ley por la cual se crearon, en 1915, las Escuelas Normales para Maestros, que surgieron con la misión fundamental de dotar de un amplio conocimiento pedagógico y cultural a los educadores que iban a ejercer en la enseñanza pública. En función de comprender la importancia de este fenómeno, es necesario recurrir a los antecedentes de estos centros escolares.

\footnotetext{
3 Algunas de las investigaciones que han tratado lo referente a la formación del magisterio son: Nicolás Garófalo, Estudio del devenir histórico de la superación de los maestros primarios de las escuelas públicas en Cuba, 1899-1958 (La Habana: Pueblo y Educación, 2008); Alicia Conde Rodríguez: "Magisterio y sociedad en una república fracturada 19001920"; Avelina Miriam Vásquez, en su tesis doctoral "Evolución histórica educacional y pedagógica del maestro primario en Cuba desde 1898 hasta 1952"; Baldomero Expósito y A. Barroto Dávila, Apuntes del movimiento de los trabajadores de la educación 18991961 (La Habana: Ciencias Sociales, 1965); Ricardo Quiza: "Cuba: historia, escuela y nacionalismo ( 1902-1930”), en Debates Americanos, No. 5-6.
} 
En el contexto de la etapa colonial se creó la primera Escuela Normal en Cuba, en Guanabacoa, en el año 1857. Aunque se creación fue un gran adelanto, pues se iniciaba en el país la formación regular del maestro de primeras letras, no cumplió con los fines esenciales para la eficacia de su enseñanza. Su labor tuvo lugar hasta 1868, pues sobrevino su clausura con el estallido del proceso independentista en la Isla. Posteriormente, la Sociedad Económica fundó una Escuela Preparatoria para maestros en 1878, en La Habana. ${ }^{4}$ Otro momento importante fue el 20 de julio de 1882, cuando se aprobó un Reglamento para las Escuelas Normales de Maestros de la provincia de La Habana, que debían ser las encargadas de formar los maestros y las maestras para las escuelas primarias de niños y de niñas de toda la isla. Su concreción tuvo lugar en 1890.

El balance preliminar realizado confirma que, en la historiografía cubana, durante años se han realizado estudios dirigidos a tratar diferentes tópicos sobre la Historia de la Educación, pero aún es necesario continuar aportando al respecto, sobre todo en lo referido a los periodos de tratamiento deficitario y las particularidades del acontecer en regiones y localidades, en especial el objeto del interés investigativo, asociado al acontecer en la ciudad de Santiago de Cuba.

\section{Metodología}

El estudio que se presenta corresponde a una investigación de carácter histórico, que utiliza métodos y técnicas del nivel teórico, como: históricológico, que favorece el estudio del comportamiento de la labor del magisterio público santiaguero según la especificidad de las coyunturas política y social del periodo, y la interpretación de forma cronológica de los hechos, con una concepción dialéctica del proceso. Análisis-síntesis, que proporciona instrumentos necesarios para una mejor comprensión del objeto de estudio, a fin de profundizar en las particularidades del fenómeno a desarrollar. Y el método hermenéutico, para el análisis e interpretación de los diferentes documentos y textos empleados en el desarrollo de la investigación.

Además, como fuentes de información, se tuvieron en cuenta las publicaciones de la prensa de la época, como los periódicos "El Cubano Libre",

${ }^{4}$ Luciano de Acevedo, "Las Escuelas Normales en Cuba", en Cuba Contemporánea, La Habana, Año 11, Tomo 32, Nº 125 (mayo, 1923): 68. 
"Ilustración Cubana" e "Instrucción Primaria", los documentos del Archivo Histórico Provincial de Santiago de Cuba, que exponen los problemas, hechos y acontecimientos del contexto histórico y su trascendencia.

\section{Planteamiento del problema}

Al tomar en cuenta la necesidad del registro de la obra del magisterio santiaguero en el periodo 1899 a 1915 en Santiago de Cuba, se pudo definir como problema científico: ¿Cuáles fueron las vías que determinaron la formación y superación del magisterio público de la enseñanza primaria en Santiago de Cuba en el periodo de 1899 a 1915 ?

La respuesta a este interrogante incita a la investigadora a profundizar en los estudios de la época. Se reconoce, en este sentido, que existen insuficientes estudios previos, de ahí la propuesta, que contribuye a consolidar la identidad profesional pedagógica del santiaguero.

\section{Resultados}

Los resultados se presentan a partir de estos indicadores:

3.1 Inicios de la formación del magisterio público. Viaje a la Escuela Normal de New Paltz.

3.2 Primeras instituciones docentes en la preparación de los maestros en Santiago de Cuba. Los exámenes de oposición.

3.3 Las Escuelas Normales de Verano: espacio para la superación del magisterio público.

\subsection{Inicios de la formación del magisterio público. Viaje a la Escuela Normal de New Paltz.}

El Superintendente de Escuelas de Cuba tuvo la misión de redactar y firmar los nuevos programas de estudio, los libros de textos, a emplearse en las escuelas públicas y los exámenes a que tendrían que someterse los maestros que desplegarían su labor en la enseñanza pública.

Una de las disposiciones que se promulgaron al efecto, con el objetivo de dotar al conjunto de hombres y mujeres a trabajar en las escuelas públicas, 
fue la Circular No. 20, del 20 de noviembre de 1900, que establecía que no era producente examinar en aquellos momentos a ese personal docente, debido a la falta de preparación y a la inexperiencia de esa gran masa de jóvenes maestros. ${ }^{5}$

Por consiguiente, se previó que, para obtener los certificados de "Primer grado", se efectuarían exámenes a partir de 1901 y solo tendrían vigencia por un año. A partir de ese momento, la realización de los exámenes, con los temas a evaluar en cada asignatura, sería el procedimiento a llevar a cabo para la selección y posterior aprobación de quienes tuvieran los requisitos que los acreditasen para integrar las filas del magisterio.

Otra de las alternativas a la que se acudió fueron los viajes a centros de educación superior en los Estados Unidos. La invitación a los docentes se dio a conocer en la Gaceta del 17 de junio de $1900 .{ }^{6} \mathrm{El}$ contenido fundamental de este proyecto consistió en que esas personas recibieran un amplio Plan de estudios que pretendiera perfeccionar su nivel cultural y metodológico, para ayudarlos en la ejecución de su futuro accionar como educadores.

A Santiago de Cuba también se extendió la convocatoria. Desde su divulgación, los maestros de esta capital mostraron interés en el asunto. Se realizaron pruebas para seleccionar a los candidatos para el viaje a suelo norteamericano, cuyo centro de estudio sería la Escuela Normal de New Paltz, en el Estado de New York. Los exámenes se desarrollaron el 24 de julio de 1901, en la Casa Escuela, ubicada en la calle de San Tadeo, No. 7. El tribunal calificador tuvo como máxima autoridad al Superintendente Provincial y otros reconocidos maestros de la ciudad. ${ }^{7}$

Posteriormente, los escogidos tendrían que enfrentarse a un tribunal central, que se constituyó en La Habana. Llama la atención que el sexo femenino fue el único que integró este proyecto en la ciudad de Santiago de Cuba. De las veinte señoritas que se presentaron, solo cuatro suspendieron. Los nombres de las que aprobaron fueron: Elisa Olozábal, Isabel Masó, Rosa Anders, Isabel Urbina, María Caro, Rafaela Infante, Esperanza Somodevilla, María J. Martínez, Carmen Cruz, María Rodríguez, Buenaventura González, Amanda Garrido, Caridad Pujols, Leocadia Araujo, Josefa F. Infante y Aquilina Canals.

5 Yoel Cordoví Núñez, Magisterio y nacionalismo en las escuelas públicas de Cuba (La Habana: Editorial de Ciencias Sociales, 2012), 9.

6 Ibíd., 21.

7 El Cubano Libre, 24, julio, 1901, 2. 
De las maestras que se seleccionaron, doce pertenecían al acreditado Centro de Instrucción de esta ciudad, encargado de la superación del magisterio. ${ }^{8}$ Algunas de estas docentes desarrollarían, poco tiempo después, importantes acciones en pos del mejoramiento de la instrucción pública primaria en el territorio, ya fuese desde su condición de educadoras, hasta directoras de los centros escolares, pero el mérito de mayor reconocimiento radicó en que, a pesar de realizar estudios en aquella nación extranjera, supieron incorporar en su accionar la defensa de la patria y, en especial, de su terruño.

La estancia de las jóvenes cubanas en suelo estadounidense fue motivo de aceptación por las autoridades del plantel docente. Así lo hizo constar una misiva que emitió el director de ese centro, Mr. Myron F. Seudden, que se publicó por la prensa de la ciudad. Así manifestó:

(...) Más de doscientas señoritas del Estado se disponen a recibir a sus condiscípulas cubanas, y coadyuvarán con nosotros a hacer agradable, amena y dichosa su visita, mientras dure su estancia entre nosotros. Deseamos que nuestras intenciones amistosas hacia las cubanas, futuras alumnas de este plantel, sean conocidas por todos los cubanos. ${ }^{9}$

Esta propuesta de enviar jóvenes cubanas al centro de estudios de Norteamérica, como parte de su superación profesional, estuvo en correspondencia con la visión que tenía sobre el asunto el entonces comisionado de Escuelas Públicas, el estadounidense Mathew E. Hanna, amigo personal de Wood. Este personaje, contrario a las ideas de algunos pedagogos cubanos de formar a los maestros en nuestro país, fue el artífice de enviar a las maestras cubanas a este curso regular en New Paltz ${ }^{10}$ (Garófalo, 2018: 39), con el que se preveía que, al retorno, las cursistas sirvieran de personal técnico de orientación y supervisión escolar.

Aun cuando la organización de los viajes dejaba entrever la posición hegemónica de los políticos norteamericanos en el plano ideológico respecto a Cuba, no se puede desestimar la importancia que tuvieron en el contexto en que se implementó. En primer lugar, porque se iba a producir

${ }^{8}$ El Cubano Libre, Año 7, $\mathrm{N}^{\circ}$ 172, 29, julio, 1901, 2. Al parecer, según revela la documentación, a la Escuela Normal de New Paltz solo iban a asistir alumnas cubanas por un tiempo de 40 semanas.

9 El Cubano Libre, 6, agosto, 1901, Año, No 179, 2.

${ }^{10}$ Nicolás Garófalo, Estudio del devenir histórico..., 39. 
el acercamiento entre los maestros cubanos, hasta ese momento aislados en sus provincias, los cuales tuvieron la oportunidad de compartir no solo sus vivencias, sino también sus futuras proyecciones, para que, cuando llegaran a su tierra natal, pudieran contribuir a la reafirmación de la cubanidad.

Un ejemplo lo constituyó el pedagogo y hombre de letras Regino Boti, quien manifestó, en su fabulosa obra testimonial Harvardianas y otros saltos al norte,

(...) que, aunque fue con sus temores al país norteño, por cuanto existía un interés político en el proyecto, el poder conocer la realidad cotidiana y la historia de ese pueblo lo conllevó a admirar y defender la suya. ${ }^{11}$

Aunque las autoridades norteamericanas pretendieron seducir a esos jóvenes cubanos en una tierra con una realidad económica diferente a la de la Isla, con el propósito de convertirlos en instrumento útil a su ideal sociopolítico, por cuanto ellos eran los encargados de transmitir esa imagen a las futuras generaciones de cubanos, buscaron como mecanismo tratar de desconocer la Historia de Cuba y de sus personalidades relevantes, pero el resultado final fue totalmente contrario a lo que se esperaba. Muestra de esto no solo fueron quienes se convirtieron en paradigmas de la pedagogía cubana, sino esencialmente de la defensa del patriotismo, como fue Ramiro Guerra, sino que muchos desde su predio: el aula, brindaron una educación dirigida al respeto y admiración de la Historia nacional, basado en el accionar y admiración de los próceres de la gesta emancipadora.

Como fundamento de esto, el profesor e historiador norteamericano Louis A. Pérez J., en su obra La estructura de la Historia de Cuba. Significados y propósitos del pasado, explica cómo el proceso de liberación del siglo XIX había actuado decisivamente en la formación de la estructura moral de la nacionalidad cubana. ${ }^{12} \mathrm{Y}$ es que la lucha por la emancipación, y sus hombres más notables, fueron objeto de preservación por parte del magisterio público, cuya expresión más visible fueron las conmemoraciones a hechos históricos y el levantamiento de estatuas en honor de un patriota, entre otras acciones.

${ }^{11}$ Regino Boti, Harvardianas y otros saltos al norte (Guantánamo: El Mar y la Montaña, 2018), 20.

${ }^{12}$ Louis A. Pérez, La estructura de la historia de Cuba. Significado y propósitos del pasado (La Habana: Ciencias Sociales, 2017), 176. 
A pesar de que en los planes imperialistas estaba utilizar la instrucción pública y a sus maestros como escalada para su proyecto anexionista, este intento no tuvo el éxito previsto, debido a la idiosincrasia de un pueblo que, tras cruentos años de conflicto bélico, había visto arraigar un sentimiento de defensa de la cubanidad; además, porque en los puestos claves de la dirección de Instrucción Pública estuvieron algunos educadores de marcada postura independentista y propugnadores del independentismo. ${ }^{13}$

Como escribiese la reconocida historiadora Marial Iglesias, en su notable obra Las metáforas del cambio en la vida cotidiana: Cuba 1898-1902

lejos de convertirse en anexionistas, muchos de estos maestros públicos regresaron a sus pueblos y ciudades persuadidos de la necesidad de convertir a su patria en una nación soberana, y de formar en esos mismos rituales a sus alumnos, en ciudadanos cubanos imbuidos del espíritu de amor y respeto a su patria. ${ }^{14}$

\subsection{Primeras instituciones docentes en la preparación de los maestros en Santiago de Cuba. Los exámenes de oposición.}

Otra de las expresiones de la gestación de esos maestros y maestras a ejercer en la enseñanza pública cubana fueron las instituciones que crearon intelectuales y pedagogos de buena voluntad, en defensa de una escuela netamente cubana. En Santiago de Cuba sobresalieron las que en seguida se señalan: la Academia que dirigió Desiderio Fajardo Ortiz, la Academia Preparatoria para maestros que asumió José Ramón Villalón, la Academia de Instrucción que fundó la maestra Ana Luisa Alayo, una Academia Nocturna para adultos "José de la Luz y Caballero", por el maestro Juan Cruz Martínez, por solo citar algunas de las más representativas durante esta etapa.

Las que dejaron la huella más imperecedera en el trabajo de preparación y superación del gremio magisterial fueron las que tutelaron dos importantes educadores: Juan Cruz Martínezy Desiderio Fajardo Ortiz. El primero, en su plantel, la Academia Nocturna José de la Luz y Caballero, se destacó en la utilización de métodos mixtos en la impartición de las asignaturas, así como contó con una valiosa Biblioteca Popular, que funcionaba en el horario de la noche, que atesoraba una

\footnotetext{
${ }^{13}$ Alejandrina Penabad, Nuestra común historia. En torno al 98 (La Habana: Ciencias Sociales, 1996), 28.

${ }^{14}$ Marial Iglesias, Las metáforas del cambio en la vida cotidiana: Cuba 1898-1902 (La Habana: Ediciones Unión, 2003), 143.
} 
valiosa colección de ejemplares pedagógicos en función de la preparación de los alumnos. ${ }^{15} \mathrm{El}$ trabajo que desempeñaron los cinco profesores del centro escolar se verificó en el éxito que tuvieron muchos de los estudiantes en los correspondientes exámenes que debieron vencer para ejercer en el magisterio público.

Desiderio Fajardo Ortiz fue clave en la defensa del sentimiento patriótico y la superación de los educadores en los albores de la República. Apodado con el calificativo "El Cautivo", fue un hombre de letras y también un relevante maestro. ${ }^{16}$ Se manifestó no solo en su postura contra el colonialismo español, lo cual determinó su ausencia de Cuba, sino en cuanto a su retorno, desplegó una labor pedagógica, de la cual surgieron representantes notables del magisterio público santiaguero.

Una de las iniciativas que tuvo fue la solicitud, el 14 de febrero de 1900, a la Junta de Educación, que cediese uno de los salones de la Academia de Bellas Artes para, en horas de la noche, establecer una Academia preparatoria de maestros de enseñanza primaria ${ }^{17}$ que, luego, se conocería como Centro de Instrucción.

En este local, los estudiantes recibieron una preparación rigurosa, en gran parte para enfrentarse al examen anual para la obtención del certificado que les permitía el desempeño de su profesión, y adquirir los aspectos medulares que, en el orden metodológico, eran necesarios para que desarrollaran su labor docente.

El accionar docente de Fajardo, en su ciudad, aunque breve, ya que comprendió los años de 1899 a 1905, fue intenso, cuyo designio fundamental fue el mejoramiento de la niñez y el magisterio, labor que tuvo el apoyo del entonces alcalde Emilio Bacardí.

Un aspecto que caracterizó el trabajo de "El Cautivo", en su Academia para maestros, radicó en que combatió las fatigosas lecciones memorísticas, rezago del pasado colonial, e incitó a propiciar un mayor uso de los medios auxiliares de enseñanza. Así lo hizo constar quien fuera uno de sus más destacados alumnos, el pedagogo Miguel Ángel Cano, continuador de su legado y que, años después, en una de sus obras, dedicó un epígrafe a su querido maestro; así lo reflejó:

(...) Las personas a quienes se podía recurrir en busca de ayuda y consejo, en busca de orientación pedagógica, eran muy pocas. En Santiago de Cuba apareció en primer término, el nombre de Desiderio Fajardo (...). ${ }^{18}$

\footnotetext{
${ }^{15}$ Alcibíades S. Poveda, Las noticias de la historia, 1902-1958 (Crónicas de Santiago de Cuba) (Santiago de Cuba: Editorial Oriente, 2015), 16.

${ }^{16}$ Edith Valls Fajardo, "Desiderio Fajardo Ortiz", en Rotaria de Santiago de Cuba. Órgano Oficial del Club Rotario, Año 6, No 4 (febrero, 1955): 14.

${ }^{17}$ AHPSC: Acta Capitular No 1 (1900): 44.

${ }^{18}$ Miguel Ángel Cano, Cuestiones pedagógicas (Santiago de Cuba: Arroyo Hermanos., 1928), 47.
} 
La ciudad de Santiago de Cuba también asumió la preparación del novel magisterio a través de los exámenes, por lo cual obtenían un certificado que los acreditaba para el desempeño en la instrucción pública primaria. Como ya se había aludido, el Superintendente General de Escuelas de Cuba ya había promulgado la Ley que autorizaba la realización de estas pruebas. No obstante, no podían efectuarse con premura, como quedó esclarecido. Se trataba de buscar los hombres y mujeres más idóneos para el desempeño de la tarea.

En este camino, les correspondió a las Juntas de Educación de cada Municipio hacer los nombramientos de aquellos maestros a ejercer en las escuelas públicas. Referente a la Junta de Santiago de Cuba, en función de cumplir con la disposición de la máxima autoridad escolar, esclareció que no se iba a exigir certificado alguno antes del $1^{\circ}$ de septiembre de 1900 , pero, luego que pasara esa fecha, todo maestro debería estar provisto de este documento. ${ }^{19}$

Esta medida estuvo a tono con la Orden Militar No. 226, del 16 de diciembre de 1899, que, entre otros asuntos, anunciaba su desarrollo y, para tales propósitos, el Superintendente de Escuelas de Cuba autorizó a las Juntas de Educación a designar, por un periodo de tiempo que no pasara del 31 de agosto de 1900, a cualquier hombre o mujer que tuviese las condiciones necesarias para instruir en las escuelas públicas.

Para retomar lo relativo a la circular No. 20, el Superintendente Provincial dio a conocer que, en primer lugar, se debía presentar la solicitud para ser aspirante a la realización de los exámenes para maestros. En este documento, tenían que consignarse estos datos: nombres y apellidos, edad, naturalidad, residencia, ciudadanía. Los exámenes se iban a efectuar en dos sesiones, de tres a cuatro horas cada una, a partir del 25 de junio de $1901 .^{20}$

En la primera sesión, se realizarían los ejercicios de las asignaturas de Historia de Cuba, Higiene, Lenguaje y Gramática, y en la segunda parte las de Gramática, Aritmética y Métodos de Enseñanza. Para la evaluación de las respectivas pruebas, se iba a conformar un tribunal con tres calificadores. Otro aspecto importante que contempló la parte organizativa de los exámenes a maestros, consistió en que aquellos que resultasen desaprobados se iban a archivar por el término de 90 días, al cabo de los cuales se desecharían, en caso que no se hubiese hecho alguna reclamación. ${ }^{21}$

${ }^{19}$ AHPSC, Gobierno Provincial. Materia Instrucción Pública, Legajo 869, (1900): 3.

${ }^{20}$ El Cubano Libre, 29, julio, 1901, 2.

${ }^{21}$ Ibid. 
Desde la realización de los primeros exámenes, se convirtieron, año tras año, en una práctica recurrente para poder desempeñarse en la enseñanza pública primaria. Como casi todos los procesos, esta actividad no estuvo exenta de críticas o alabanzas en la sociedad. De todas maneras, si hubiera un elemento a destacar desde su concepción, radica en que su objetivo esencial fue que los maestros sintieran la necesidad de estudiar y, por ende, superarse para el logro fructífero de su quehacer profesional y, además, que pudieran conservar su puesto de trabajo.

Referente a los señalamientos que se le imputaron, se considera que la esencia estuvo en que se aprobase una Ley, por la cual se instituyesen las Escuelas Normales para la formación regular del magisterio: a tono con este reclamo, algunos intelectuales manifestaron su inquietud respecto a la urgencia que existía de crear esa institución pedagógica, en pos de una preparación más adecuada a aquellos hombres y mujeres (Ilustración Cubana, 1906: 6); sin lugar a dudas, esta idea fundamentaba que los exámenes mantuvieron en una constante presión al magisterio.

Pero si bien hubo quienes coincidieron con el reclamo, cada una de las pruebas a efectuarse y sus contenidos, hubo otros que aplaudieron su creciente rigurosidad. Un ejemplo lo constituyó la solicitud que el 5 de mayo de 1907 emitiera un grupo de maestros públicos, dirigidos por el educador José Ramón Villalón, al entonces Superintendente Provincial, cuya esencia radicó en la realización de los exámenes correspondientes a ese año, en que "no imperase la benevolencia excesiva de años anteriores", ante cuyo reclamo, supuestamente inapropiado, y en respuesta a las facultades que le confería el cargo, el Superintendente Rafael Odio Savala expresó:

(...) las pruebas que se desarrollaron estuvieron en consecuencia con las exigencias de la Ley, así como también que aumentar el rigor resultaría contraproducente para los aspirantes. ${ }^{22}$

Luego de manifestar su posición respecto al asunto, Odio enfatizó que lo que sí era de obligatorio cumplimiento para todos los aspirantes, y que no era motivo de cambios, era cumplir con los parámetros establecidos al efecto, como:

${ }^{22}$ Ibid. 
1) Prohibido llevar al acto del examen libros, cuadernos o apuntes.

2) Conversar durante el desarrollo del ejercicio.

3) Hacer señalizaciones en los trabajos, que pudieran revelar el nombre del autor (Ídem).

Por consiguiente, si bien los ejemplos anteriores constituyeron una muestra de las opiniones divergentes sobre la realización de los exámenes, se efectuaron de manera sistemática hasta la creación de las Escuelas Normales. Cada año, la prensa de la ciudad, esencialmente el periódico El Cubano Libre, ofrecía, a través de sus páginas, especialmente en la casi ineludible sección "Instrucción Pública", las convocatorias para los solicitantes a integrar las filas del magisterio público. Con este método, los maestros tuvieron que prepararse para el desempeño de su labor, en que, dada la cantidad de materias en las cuales tenían que examinarse, se considera que sí hubo rigor en la ejecución de estas pruebas.

Estas actividades se desarrollaron casi siempre en dos a tres días, debido a lo extenso del programa, y el Tribunal se integró por miembros respetables del magisterio de la ciudad y por autoridades escolares. Luego de su culminación y revisión, los resultados casi siempre se daban a conocer en el local de la Junta de Educación o a través de la prensa. Como en todo proceso en procura de mejorar, desde su implementación, cada año se trataba de lograr niveles mayores en el orden cualitativo y en su organización.

También, la prensa devino un espacio por excelencia, en el cual autoridades del sector, intelectuales y notables maestros, publicaron artículos que orientaron y sirvieron para continuar preparando a ese claustro para ejercer en las escuelas públicas primarias, cuando todavía el surgimiento de las Escuelas Normales era un reclamo de aquellos que sabían sobre la importancia que tenían para una formación acertada y regular de aquel magisterio.

\subsection{Las Escuelas Normales de Verano: espacio para la superación del magisterio público.}

No obstante, al no existir en los albores de la República cubana una institución docente que tuviera la responsabilidad de formar curricularmente a los hombres y mujeres para ejercer en el magisterio, y al tener que enfrentarse a 
exámenes de oposición para avalar su condición como docente, se necesitó la superación de esos maestros. Reconocidos intelectuales, como Arturo R. Díaz, director de la revista La Escuela Moderna, de carácter pedagógico; Enrique José Varona, secretario de Instrucción Pública, fueron partidarios de establecer en cada capital de provincia, en Cuba, las Escuelas Normales de Verano; es decir, instituciones que contribuyeran a la preparación de los maestros del mañana.

Desde su surgimiento en 1900, estos establecimientos escolares funcionaron entre los meses de julio y agosto. Al llamado de este proyecto, netamente cubano, acudieron profesores universitarios y de segunda enseñanza, escritores, en esencia, hombres de gran cultura y reconocimiento en el mundo de la intelectualidad. Por vez primera, los pedagogos y profesionales de renombre tuvieron la posibilidad de transmitir sus conocimientos docentes y culturales, por el bien de aquellos maestros jóvenes en plena formación; y que tenían la magna responsabilidad de instruir y educar a las generaciones del mañana, con una franca vocación patriótica. Por ende, se afirma que dichas instituciones se justificaron, por la necesidad de orientar a los noveles docentes hacia nuevos rumbos y distintos a los seguidos hasta entonces.

Santiago de Cuba también fue escenario de la creación de este tipo de centro docente. En fecha tan temprana como el 19 de diciembre de 1899, la Asociación de Profesores Públicos de esta Provincia envió una comunicación al secretario de Justicia e Instrucción, entre cuyas demandas estuvo "la creación de una Escuela Normal para que formase maestros a la altura de la situación, cerrando las puertas a gente extraña que busca la enseñanza como modo de vivir". ${ }^{23}$

Esta idea se vio materializada el 14 de julio de 1902, al inaugurarse, en la Escuela Pública no. 12, la Escuela Normal de Verano del Distrito Escolar, bajo la presidencia del Superintendente de Instrucción Manuel Navarrete. Como todas las creadas en territorio nacional, tendría una Escuela Práctica Anexa, cuyo mérito residía en que los alumnos podrían aprender sobre los métodos de enseñanza en boga, y su ejercitación sería con los niños presentes, discípulos que no solo recibieron los contenidos de manera teórica, sino tuvieron la posibilidad de aplicarlo, lo cual les permitió una mejor aprehensión de los contenidos.

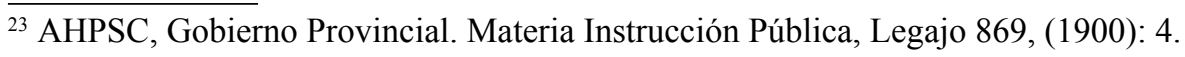


En la antigua provincia de Oriente se establecieron tres escuelas de Verano: una en Santiago de Cuba, a la que se adscribieron el Distrito urbano de esta ciudad y los municipales del Caney, Cobre, Jiguaní, Palma Soriano, San Luis y Songo; otra en Holguín y la otra en Manzanillo. ${ }^{24}$

En el caso de la que se instauró en Santiago de Cuba, desde los inicios contó con un claustro de excelencia, por cuanto participó un reconocido grupo de hombres y mujeres, que poseían no solo la experiencia, sino, también, el bagaje intelectual necesario para el desempeño de esta importante tarea. Sus nombres: Desiderio Fajardo Ortiz, quien impartió las asignaturas de Lenguaje y Gramática y Organización Escolar; José Ramón Villalón, Aritmética; Francisca Tintoré, Lectura; Luis Cuza, Geografía; Amanda Garrido, Historia; el Dr. Ambrosio Grillo, Estudios de la Naturaleza; el Dr. Lorenzo Comas, Higiene Escolar y Ejercicios Físicos y Enrique Jardines, Métodos de Enseñanza. La Escuela Práctica la dirigió Concepción Caignet. Además, integraron el claustro, en la categoría de profesores auxiliares, las siguientes personalidades: Carmen García Pullés, María Caro, Manuela Miyares, Hipólito Jáuregui, Rosa Cause y Luis G. Navarro. ${ }^{25}$

Un aspecto positivo de estos cursos de preparación para los maestros públicos desde un primer momento fue fomentar, a través de la instrucción, el amor a la Historia patria. En función de esta preparación, se invitó a determinados conferencistas a dar lecciones sobre la temática; en este sentido, se significa la clase que impartió el Dr. Fernández Marcané sobre contenidos de Historia de Cuba en 1901; al concluir su disertación, se dirigió al auditorio para exhortar a los educadores a que impulsasen de manera constante en sus alumnos el patriotismo y la recordación de los héroes de la gesta independentista, por ejemplo, la figura de José Martí. ${ }^{26}$

Las Escuelas Normales de Verano, con el transcurrir de los años, se fueron perfeccionando; por ejemplo, la Superintendencia Provincial de Escuelas de Santiago de Cuba, en el curso de 1902 -1903, dedicó sus esfuerzos a hacer de las Escuelas Prácticas anexas a las Normales el centro rector del funcionamiento de estos centros; en esencia, tributarían con mayor énfasis a que los maestros realizaran trabajos prácticos en el aula.

Estas instituciones docentes sufrieron algunas transformaciones en su labor, hasta su desaparición en 1909. Y para profundizar en esto, se tiene

${ }^{24}$ Instrucción Primaria, Año 2, No 9, 10, diciembre, 1903: 297.

${ }_{25}$ Instrucción Primaria, Año 2, No 9, 10, diciembre, 1903: 297.

${ }^{26}$ El Cubano Libre, 26, enero, 1901: 2. 
que, a partir del año 1906, cuando el Partido Moderado impulsó la reelección del entonces presidente Tomás Estrada Palma, frente a la candidatura liberal de José Miguel Gómez y Alfredo Zayas, ante la imposición moderada, los liberales acudieron a la violencia, en la conocida Guerrita de Agosto del propio año. Ambos bandos coincidieron en buscar la solución en el vecino del norte. Fue así como se produjo la segunda intervención norteamericana. Ahora, el nuevo gobierno buscaría las fórmulas que le permitieran restablecer los mecanismos institucionales para el logro de la necesaria estabilidad. En ese contexto, le correspondió a la figura de Charles Magoon aplicar las leyes para el logro de los mencionados objetivos. En esta nueva coyuntura, el sector educacional fue escenario de algunos cambios que previeron las autoridades norteamericanas.

Una comisión de maestros de La Habana, integrada por Leopoldo R. Tamayo, Ismael Clark y Edito Aparicio, se entrevistó el 28 de abril de 1907 con el gobernador provincial para solicitar la modificación del Plan de exámenes y establecer algunas reformas en las Escuelas Normales de Verano. ${ }^{27}$ Entre los cambios que se discutieron, estuvo que a dichas instituciones no las financiaran por cuenta del Estado, lo cual se notificó a las escuelas primarias de la Provincia de Oriente. Otro aspecto que se abordó fue la eliminación de la cuota que tenían que abonar los maestros para cursar sus estudios en estos centros educacionales $\mathrm{Y}$ se mantuvo la obligatoriedad de la asistencia a clases. Los casos excepcionales se analizarían por la Junta de Educación, para su aprobación o no.

Si bien estas medidas apuntaban esencialmente al aspecto organizativo de estos centros docentes, los cambios más importantes que se esperaban tenían que dirigirse a la elevación cada vez mayor de una mejor calidad en la docencia. Por consiguiente, se planteó que el claustro asumía la misión de formar a esos jóvenes, que tenían que ser mayoritariamente provenientes del sector magisterial, por supuesto con una fructífera labor intelectual. En aquellos momentos, este asunto era necesario por cuanto, en sus inicios, las Escuelas Normales de Verano tuvieron que acoger a muchos profesionales de diversas especialidades.

No obstante, al igual que ocurrió en esos primeros momentos, y sería una práctica, no se les iban a cerrar las puertas a aquellos intelectuales que no tenían una formación pedagógica, pero contaban con el conocimiento

${ }^{27}$ Ibid. 
adecuado de una conducta social favorable para que los aceptaran como conferencistas. En el nuevo contexto de cambios para poder garantizar estos requisitos, la directiva de la institución tendría la responsabilidad de contar con un trabajo previo, para conocer cuáles eran los aspectos claves que, en el orden del contenido y metodológico, trataría este profesional en su clase.

En medio de estas transformaciones, las sesiones de la Escuela Normal de Verano correspondientes al curso de 1906-1907 transcurrieron con una tranquilidad importante, amén de la coyuntura política que estaba viviendo la nación. En la nueva edición, fungieron como conferencistas ilustres personalidades, tales como: Ángeles Ramírez de Martínez, Enrique Jardines, Dr. Ambrosio Grillo, Luis Cuza, Peña y Reinoso, entre otros ejemplos. A decir de sus alumnos, "durante las cuatro semanas que duraron los cursos, hubo un ambiente de notable fraternidad entre todos los participantes". ${ }^{28}$ Así, las materias como Gramática, Geografía, Aritmética, Lenguaje, Instrucción Moral y Cívica, que explicaron de manera adecuada los profesores. El ideal pedagógico no estuvo presente en la impartición de estas lecciones; sin embargo, fue idea del claustro que las ideas a tratar en clases se expusieran en la forma más clara, precisa y transparente posible.

En sus años de existencia, las Escuelas Normales de Verano cumplieron con el buen propósito de elevar el nivel cultural de sus estudiantes y de proyectarlos con los métodos de enseñanza en boga. Durante los meses de julio y agosto, los maestros se reunían y tenían la posibilidad no solo de prepararse, sino de socializar sus experiencias, ${ }^{29}$ pero también fue el momento de la comprensión de muchos de conocer y admirar la Historia de la nación, sus héroes, y el respeto de las instituciones jurídicas, cuyo contenido lo estudiaron en las clases de Cívica y Moral, asignaturas que recibieron quienes integraron el proyecto de superación a centros universitarios de los Estados Unidos, pero que ahora tendrían la oportunidad de aprenderlas, para, luego, enseñarlas a sus pequeños alumnos.

Aun cuando muchos de estos profesionales contaron con un limitado conocimiento en temas pedagógicos, lo que en determinados momentos conllevó que las lecciones tuvieran un cargado matiz teórico, en nada disminuyó la esencia para la que se crearon estos centros docentes, que fue

28 Ilustración Cubana, Año 1, No 23, Santiago de Cuba, 1º, septiembre, 1906: 5-6.

${ }^{29}$ Ramiro Guerra, "El general Leonardo Wood y la instrucción pública". En Revista Cuba Contemporánea, Año 7, tomo 23, No 91 (1920): 201. 
la superación del magisterio. Estos conferencistas, en su afán de brindar un adecuado conocimiento y que llegase lo más didácticamente posible a sus alumnos, tuvieron que estudiar los aspectos básicos referentes a los métodos y procedimientos más adecuados para ofrecer cómo se ha planteado, de forma más eficaz, el gran caudal de conocimientos que poseían.

Aun con los resultados positivos en sus nueve años de existencia, estos planteles escolares presentaron algunas limitaciones, que llevaron finalmente a su clausura. Al respecto, el intelectual Manuel García Falcón expuso, en una de las publicaciones periódicas especializadas en materia pedagógica, algunos de los aspectos que, al cabo de unos años, tornaban inoperantes las Normales de Verano. Enfatizó que

el funcionamiento de estos centros docentes, viene a continuación de un curso escolar de nueve meses, donde la tensión nerviosa ha sido constante, y la época del año la menos propia para continuar un largo ejercicio mental. ${ }^{30}$

Se refería a lo fatigosas que resultaban muchas veces para los alumnos aquellas extensas conferencias. En busca de soluciones ante ese contexto, algunos especialistas eran del criterio que se debía impartir una sola en el día.

Aun cuando en 1909 estas instituciones docentes dejaron de funcionar, la prensa de la época, a través de secciones que se dedicaron a tópicos educativos, asumió la tarea de publicar trabajos que contribuyeran a la superación de los maestros en sus exámenes de oposición. En el caso de Santiago de Cuba, sobresalieron en ese periodo entre 1899 a 1915 El Cubano Libre y La Independencia, en cuyas páginas escribieron reconocidos pedagogos e intelectuales de la ciudad, que brindaron sus conocimientos para el mejoramiento de la enseñanza. Fue en el año de 1915 cuando se aprobó por el Congreso la creación de las Escuelas Normales para Maestros y Maestras en el país, momento a partir del cual representó un salto de calidad para la escuela pública cubana.

${ }^{30}$ Manuel García, “Las Escuelas de Verano”. En Revista Cuba Pedagógica, Año 5, № 28, 30, mayo, 1907: 2-3. 


\section{Conclusiones}

La formación del magisterio público a desempeñarse en la instrucción pública primaria en la ciudad de Santiago de Cuba estuvo a tono con las disposiciones que a nivel nacional las autoridades norteamericanas llevaron a cabo para el sector. En consecuencia, y como parte de su estrategia ideo política, se concibieron viajes a centros universitarios de Harvard y la Escuela Normal de New Paltz, en virtud de prepararlos para el futuro ejercicio de su profesión. En el escenario santiaguero, varias maestras acudieron al llamado que formularon las autoridades escolares para que cursaran estudios en la Escuela Normal de New Paltz.

Otra de las vías de formación del magisterio público fueron los exámenes de oposición que tuvieron que realizar los hombres y mujeres que quisieran desempeñarse en las escuelas primarias públicas, proceso después del cual se les otorgaba un certificado que avalaba el poder ejercer la profesión. Ellos fueron los llamados "maestros de certificado", procedimiento que se efectuó hasta la constitución de las Escuelas Normales para Maestros que, en el caso de Santiago de Cuba, tuvo lugar en el año 1916.

Si bien estas vías constituyeron los primeros pasos en la preparación inicial de los maestros, un elemento que tuvieron presente las autoridades escolares fue la superación de un claustro joven, carente todavía de las herramientas necesarias para el ejercicio satisfactorio de su labor, ante la inexistencia, en los albores de la República, de una institución que los dotara de una formación curricular apropiada y con carácter regular. En esta dirección vio la luz la Escuela Normal de Verano en el territorio de Santiago de Cuba, la cual se creó en 1902, y que en sus pocos años de existencia contó con un grupo de intelectuales y pedagogos que brindaron sus conocimientos para los educadores del nivel primario.

Otra de las expresiones que contribuyó al mejoramiento como docentes fue la relativa a las instituciones educativas que fundaron destacados pedagogos del territorio, cuyo objetivo esencial era brindar las herramientas no solo del contenido, sino también metodológicas, para que los futuros maestros del nivel primario pudieran enfrentarse con calidad a los exámenes de oposición. En Santiago de Cuba, sobresalió la Academia que dirigió Desiderio Fajardo Ortiz, uno de los representantes del gremio magisterial más connotados de los primeros años republicanos. 
La formación y superación de los hombres y mujeres que se desempeñaron en las escuelas primarias públicas estuvo en la agenda primeramente de las autoridades norteamericanas de ocupación y, luego, de los gobernantes cubanos, una vez que se constituyó la República en 1902. La necesidad imperante del logro de un docente de calidad determinó que diferentes voces del ámbito magisterial e intelectual se pronunciaran por la creación de una institución rectora de los estudios pedagógicos en Cuba, es decir, las Escuelas Normales para Maestros. Previo a este momento, y aun con las limitaciones educativas, didácticas, la primera hornada de maestros en Cuba y, en el caso que nos ocupa, de Santiago de Cuba, se proyectó por brindar una educación de calidad y de gran sentir patriótico a las nuevas generaciones.

\section{Referencias}

AHPSC. Acta Capitular, No. 1. 1900.

AHPSC, Acta Capitular, No. 3.1902.

AHPSC, Gobierno Provincial. Materia Instrucción Pública, Legajo 869. 1900.

AHMSC, Gobierno Municipal. Fondo Asuntos Patrióticos, Legajos 20-56.

Boti, Regino E. Harvardianas y otros saltos al norte. Guantánamo: El Mar y la Montaña, 2018.

Cano, Miguel Ángel. Cuestiones pedagógicas. Santiago de Cuba: Arroyo Hermanos, 1928.

Cordoví, Yoel. Magisterio y nacionalismo en las escuelas públicas de Cuba. La Habana: Ciencias Sociales, 2012.

Fajardo, Edith. “Desiderio Fajardo Ortiz”. Revista Rotaria, Año 6, № 4 (1955).

García, Manuel. Las Escuelas de Verano. Revista Cuba Pedagógica, Año 5, N ${ }^{\circ}$ 28 (30, mayo, 1907).

Garófalo, Nicolás. Estudio del devenir histórico de la superación de los maestros primarios de las escuelas públicas en Cuba 1899-1958. La Habana: Pueblo y Educación, 2018. 
Guerra, Ramiro. "El general Leonardo Wood y la instrucción pública". Revista Cuba Contemporánea, Año 7, Tomo, 23, № 91 (julio, 1920)

Iglesias, Marial. Las metáforas del cambio en la vida cotidiana: Cuba 1898-1902. La Habana: Ediciones Unión, 2003.

Pérez, Louis A. La estructura de la historia de Cuba. Significado y propósitos del pasado. La Habana: Ciencias Sociales, 2017.

Periódico El Cubano Libre, 26, enero, 1901.

Periódico El Cubano Libre, 3, junio, 1907.

Periódico El Cubano Libre, 24, julio, 1901.

Periódico El Cubano Libre, 29, julio, 1901.

Periódico El Cubano Libre, 6, agosto, 1901.

Periódico Ilustración Cubana, Santiago de Cuba, Año 1, No 17, 21, julio, 1906.

Periódico Instrucción Primaria, Año 2, No 9, 10, diciembre, 1903.

Periódico Ilustración Cubana, Santiago de Cuba, Año 1, Nº 23, 5-6, $1^{\circ}$, septiembre, 1906.

Periódico Ilustración Cubana, Santiago de Cuba, Año 1, Núm. 17, 6, 21, julio, 1906.

Periódico Instrucción Primaria, Año 2, No 9, 10, diciembre, 1903.

Periódico Ilustración Cubana, Santiago de Cuba, Año 1, № 23, 5-6, $1^{\circ}$, septiembre, 1906.

Penabad, Alejandrina. Nuestra común historia. En torno al 98. La Habana: Ciencias Sociales, 1996.

Poveda, Alcibíades S. Las noticias de la historia, 1902-1958 (Crónicas de Santiago de Cuba). Santiago de Cuba: Editorial Oriente, 2015. 


\section{Citar este artículo}

Mancebo Céspedes, Daineris. "El Magisterio público de la ciudad de Santiago de Cuba: formación y superación entre 1899 a 1915". Revista Historia de la Educación Colombiana. Vol. 25 No 25 (2020): 95-116. DOI: https://doi.org/10.22267/rhec.202525.80 